\title{
Recent MicroBooNE results
}

\author{
Andrew Furmanski*i \\ University of Manchester \\ E-mail: andrew.furmanski@manchester.ac.uk
}

MicroBooNE (the Micro Booster Neutrino Experiment) is a liquid argon time-projection chamber placed in the Booster Neutrino Beam at Fermliab. The physics goals of MicroBooNE are to investigate the MiniBooNE observed electron-like low energy event excess, to investigate neutrinoargon cross sections, and to develop and demonstrate technologies required for future large TPCs such as DUNE [2]. After one year of taking data, MicroBooNE is making significant progress on all these fronts. Here we present several new results, including a Michel electron energy spectrum, a fully automated $v_{\mu}$ CC-inclusive selection, and a selection of neutral pions in neutrino beam data.

Neutrino Oscillation Workshop

4 - 11 September, 2016

Otranto (Lecce, Italy)

\footnotetext{
*Speaker.

†n behalf of the MicroBooNE collaboration
} 


\section{MicroBooNE and the SBN program}

MicroBooNE is a 170t liquid argon time-projection chamber (LArTPC), situated in the booster neutrino beam (BNB) at Fermilab[1]. Roughly 90t of liquid argon are contained within the TPC itself. MicroBooNE is the first detector to be built as part of the short baseline neutrino (SBN) program at Fermilab. It will be joined in 2017 by a new short baseline near detector (SBND), and a refurbished ICARUS detector which will be shipped from Europe as a far detector.

The primary goals of MicroBooNE are to investigate the observed MiniBooNE low-energy excess of electron-like events, study neutrino interactions on argon, and develop and demonstrate various technologies required for scaling a LArTPC to the size that DUNE will require.

MicroBooNE took its first data run in 2015-2016, during which it collected over $3.4 \times 10^{20}$ protons on target (POT). Currently only $0.5 \times 10^{20}$ POT of neutrino beam data has been analysed.

\section{Michel energy spectrum}

Being situated on the surface, with minimal overburden, MicroBooNE is exposed to a very high cosmic ray flux. This allows us to identify a high statistics sample of stopping cosmic muons, and identify their decay electrons. We use these electrons to measure a Michel electron energy spectrum, which is an important calibration source.

Michel electrons are selected using a 2D clustering algorithm in the collection plane only, and searching for both a muon Bragg peak, and a visible "kink" at the decay point. The Michel energy is then corrected for electron lifetime effects, and converted into units of $\mathrm{MeV} / \mathrm{c}^{2}$ using known electronics gains. Figure 1 shows the reconstructed Michel energy spectrum. Two effects can be seen here. The first is that there are events past the normal end-point, usually due to merging some of the muons Bragg peak into the electron cluster. The second is that the peak energy is lower than may be expected. This shift is due to missing charge due to radiative losses from the electron which haven't been clustered with the electron. These effects are seen in both data and MC giving us confidence that our detector is responding in simulation the same way as in experimental data. For more details on this analysis, see [3].

\section{CC-inclusive event selection}

A critical step on the road to a test of the MiniBooNE low energy excess, and to any CCexclusive cross section measurement, is to understand the dominant event type in MicroBooNE $v_{\mu}$ CC-inclusive. For this, two selections were developed. The first (referred to as selection I), intended to be simple, restricts itself to only fully contained interactions. The second selection (referred to as selection II) allows uncontained events but only for events with more than one track. The selection then proceeds separately for different multiplicities, placing cuts specifically designed to eliminate cosmic-induced backgrounds. Both selections are preceded by a requirement that there be an optical flash of greater than 50 photoelectrons $(\mathrm{PE})$ in coincidence with the arrival of the neutrino beam.

The largest challenge is the removal of the above mentioned cosmic rays, which outnumber neutrino interactions by around 3 orders of magnitude. We therefore apply the selection on both 


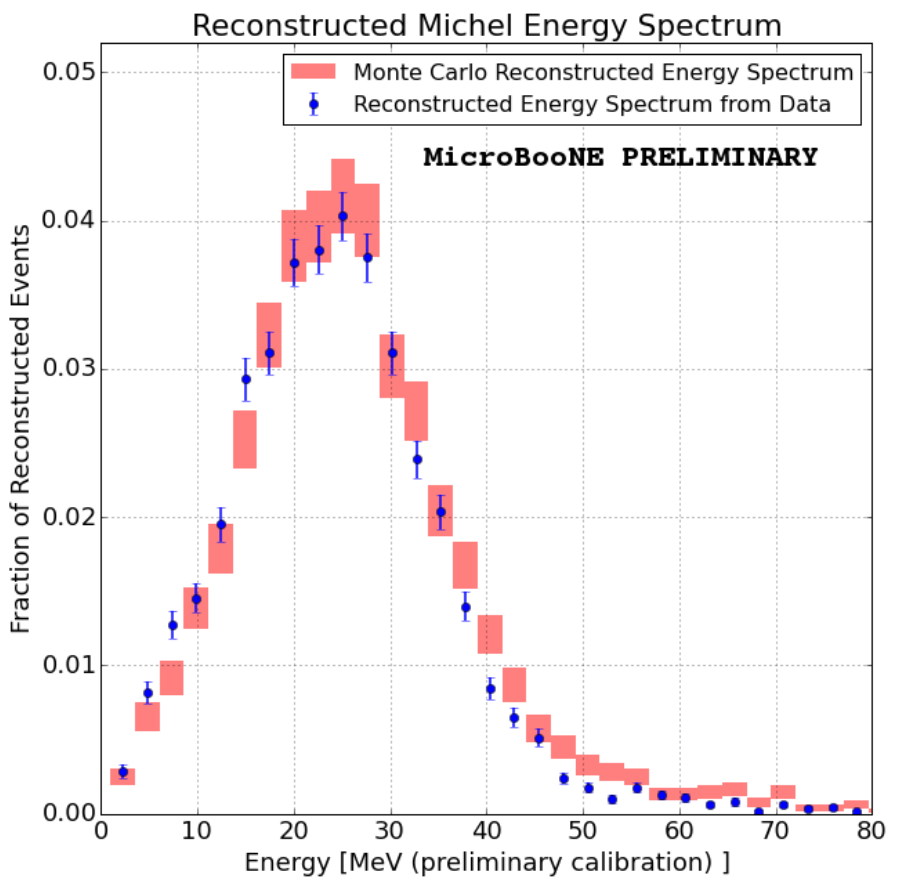

Figure 1: Reconstructed Michel energy spectrum from MicroBooNE cosmic data and MC. Statistical errors only shown.

beam data, and off-beam data, and subtract the off-beam data to remove beam-uncorrelated cosmic backgrounds. Some cosmic background remains, due to events where a real neutrino interaction leads to a large enough flash within the beam-spill, but a cosmic track within the event is selected as the neutrino candidate. This background is estimated with Monte Carlo.

Figure 2 shows the muon momentum and angle distributions from selection II, for the $0.5 \times$ $10^{20}$ POT of data used in this study, with the beam-uncorrelated background subtracted using offbeam data. This is compared to MC with beam events and cosmic ray tracks in the same events. The MC distribution is normalised to the same number of events as the data for a shape-only comparison, and the uncertainties shown are statistical only. For more details on this analysis, including distributions from selection I, see [4].
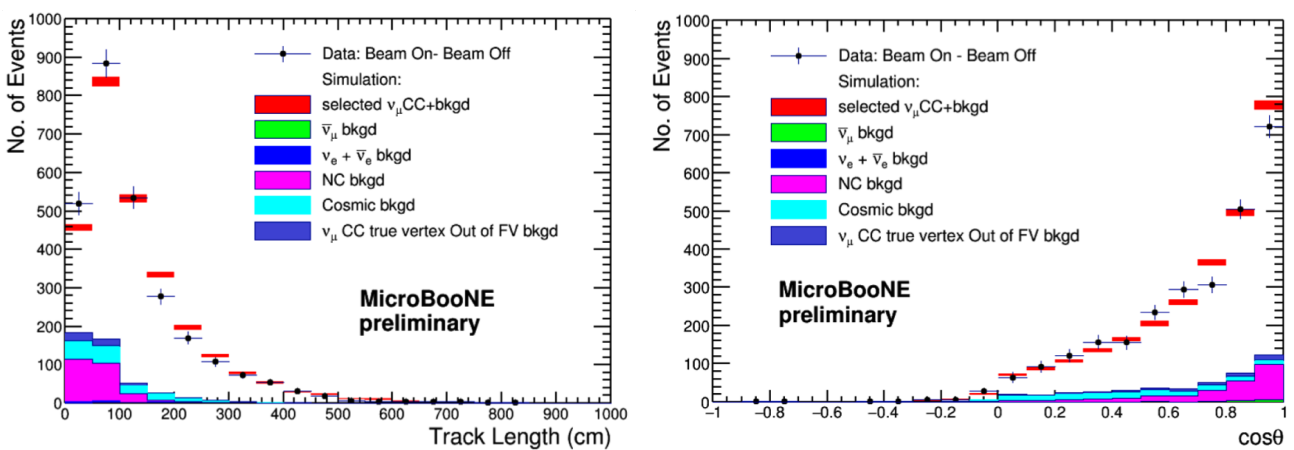

Figure 2: Selection II muon track length and angle distributions for data (with beam-uncorrelated backgrounds subtracted) and MC. The MC is normalised to the data. The error bars are statistical only. 


\section{Neutral pion selection}

A critical calibration and control sample for MicroBooNE's low energy excess search is decays of beam-produced neutral pions. The photons from these decays allow us to understand the reconstruction and calorimetry of $\sim 100 \mathrm{MeV}$ showers, and help understand the electron/photon separation from $\mathrm{dE} / \mathrm{dx}$ measurements. In addition there is expected to be a background to the electron-like signal from neutral pions where one decay photon is missed, so understanding how often this happens is very important.

As a first step, we take events that have been identified as $v_{\mu} \mathrm{CC}$ events by the previous event selection, and search for pairs of reconstructed photons near the interaction vertex. Several techniques were attempted, including reconstruction in only one view (2D), reconstruction using all three views simultaneously (3D), and pattern recognition (using a neural network). This found a high-purity sample of neutral pions in data, with reconstructed invariant masses consistent with a neutral pion in each case. For more details on this analysis, see [5]

\section{Conclusions}

MicroBooNE has collected over $3 \times 10^{20}$ POT in its first year of running. With the first $0.5 \times$ $10^{20}$ POT of this data we have demonstrated effectively the ability to measure neutrino induced muon kinematics, achieving good agreement with simulation, and the identification of neutrinoinduced neutral pions in data. These are critical steps on the path to a search for the MiniBooNE low energy electron-like excess of events.

\section{References}

[1] The MicroBooNE collaboration The MicroBooNE Technical Design Report, 2012, http://www-microboone.fnal.gov/publications/

[2] R. Acciari et al., Long-Baseline Neutrino Facility (LBNF) and Deep Underground Neutrino Experiment (DUNE), arXiv:1512.06148, 2015

[3] The MicroBooNE collaboration Michel Electron Reconstruction Using the MicroBooNE LArTPC Cosmic Data MICROBOONE-NOTE-1008-PUB, 2016

[4] The MicroBooNE collaboration Selection and kinematic properties of $v_{\mu}$ charged-current inclusive events in 5 E19 POT of MicroBooNE data MICROBOONE-NOTE-1010-PUB, 2016

[5] The MicroBooNE collaboration Demonstration of 3D Shower Reconstruction on MicroBooNE Data MICROBOONE-NOTE-1012-PUB, 2016 